\title{
Efficacy of combined immunotherapy with Propes and Inflamafertin in selective deficiency of NK and NKT cells in children with autism spectrum disorders associated with genetic deficiency of the folate cycle
}

\author{
Dmitry Maltsev', Volodymyr Stefanyshyn²
}

${ }^{1}$ Research Institute of Experimental and Clinical Medicine, Bogomolets National Medical University, Kyiv, Ukraine ${ }^{2}$ Vivere Neuroimmunology Clinic, Kyiv, Ukraine

\begin{abstract}
Objectives. The results of previous small clinical trials indicate the potential benefit of combination immunotherapy with Propes and Inflamafertin to compensate for NK and NKT cell deficiency due to genetic deficiency of the folate cycle in children with autism spectrum disorders. The purpose of the research was to study the effectiveness of combined immunotherapy with Propes and Inflamafertin in NK and NKT cell deficiency in children with autism spectrum disorders associated with genetic deficiency of the folate cycle.

Material and methods. This single-center, prospective, controlled, nonrandomized clinical trial included 96 children aged 2 to 10 years with autism spectrum disorders associated with a genetic folate deficiency (study group, $\mathrm{SG}$ ). Children of SG received Propes at a dose of $2 \mathrm{ml}$ IM every other day for 3 consecutive months (45 injections), and Inflamafertin at a dose of $2 \mathrm{ml}$ IM every other day for 3 months in a row, alternating with Propes (45 injections). The control group (CG) consisted of 32 children of similar age and gender distribution who suffered from autism spectrum disorders associated with genetic deficiency of the folate cycle, but who did not receive immunotherapy. Outcomes. The number of NK cells reached the lower limit of normal in 39 out of 53 patients ( $74 \%$ of cases), with the resulting deficiency of these lymphocytes, and the average number of NK cells in the blood in SG almost doubling during the 3-month course of immunotherapy $(p<0.05 ; Z<Z 0.05)$. However, it returned to almost initial level in the 2 months following the discontinuation of immunotherapeutic agents ( $p>0.05 ; Z>Z 0.05$ ). The number of NKT cells was normalized in 78 out of 87 patients ( $89 \%$ of cases) with an initial deficiency of these cells, and the average number of NKT cells in the blood in the DG increased during the course of immunotherapy by half $(p<$ $0.05 ; Z<Z 0.05)$ and continued to grow for the next 2 months after the discontinuation of immunotropic drugs $(p<$ $0.05 ; Z<Z 0.05)$. There was a link between immunotherapy and normalization of NK - $(X 2=18.016$; OR = 13.929; $95 \% \mathrm{Cl}=3.498-55.468)$ and NKT-cells $(X 2=60.65 ; \mathrm{OR}=46.800 ; 95 \% \mathrm{Cl}=14.415-151.937)$ in the blood with a strong association between these processes (criterion $\varphi=0.504$ and 0.715 respectively; $C=0.450$ and 0.581 respectively).

Conclusions. Combination immunotherapy with Propes and Inflamafertin is an effective strategy for the treatment of immunodeficiency caused by genetic deficiency of the folate cycle in children with autism spectrum disorders.
\end{abstract}

Keywords: autoimmune complications, immunoprophylaxis, immunomodulation, cell immunodeficiency

\section{INTRODUCTION}

Data from recent meta-analysis of randomized controlled clinical trials indicate an association between autism spectrum disorders and genetic folate deficiency in children $[1,2]$. It is established that the genetic deficiency of the folate cycle affects the immune status of children with autism spectrum disorders, forming a kind of immunodeficiency, which is based on a decrease in the number and functional activity of natural killers (NK cells) and 
natural killer T cells (NKT cells) [3]. Immunosuppression due to genetic deficiency of the folate cycle mediates the development of a number of immune-dependent complications that determine the formation of inflammatory encephalopathy in children with autism spectrum disorders, in particular - reactivated opportunistic infections [4,5], autoimmune reactions against neurons and myelin $[6,7]$ and systemic inflammation with cytokine release syndrome with hypercytokinemia $[8,9]$. The compensation of immunodeficiency, induced by genetic deficiency in the folate cycle, appears to be an attractive prospect for preventing or at least alleviating the manifestations of associated immune-related complications that affect the severity of CNS damage in children with autism. However, such therapeutic approaches remain undeveloped and are therefore not available to patients. The results of previous small clinical trials indicate the potential benefit of combined immunotherapy with Propes and Inflamafertin to compensate for the deficiency of NK- and NKT-cells in folate cycle deficiency $[10,11]$. However, this encouraging data should be validated in larger controlled clinical trials with greater weight of the results obtained. Propes - a biological agent containing alpha- and beta-defensins, has a pronounced immunoactivating and lymphoproliferative effect. At the same time, Inflamafertin, which contains alarmines and adrenomedulin, in contrast has an anti-inflammatory effect that is mediated by interleukin 10 , which is important in preventing autoimmune complications in drug-induced immune activation. Based on the experience of usage of another highly active immunomodulatory agent - recombinant interleukin 2 - therapeutic immune activation can cause an undesirable increase in the risk of autoimmune complications [12]. Therefore, the combination of immunoactivating drug Propes with an anti-inflammatory tolerogenic immunotropic agent seems to be the key to achieving safe immunomodulatory therapeutic effect.

\section{PURPOSE}

To study the effectiveness of combination immunotherapy with Propes and Inflamafertin in NK and NKT cell deficiency in children with autism spectrum disorders associated with genetic deficiency of the folate cycle.

\section{METHODS}

This single-center, prospective, controlled, nonrandomized clinical trial included 96 children aged 2 to 10 years with autism spectrum disorders associated with genetic folate deficiency. These patients formed the study group (SG). The diagnosis of autism spectrum disorders was made by psychiatrists of regional hospitals or specialized departments according to the criteria of DSM-IV-TR (Diagnostic and Statistical Manual of mental disorders) and ICD-10 (The International Statistical Classification of Diseases and Related Health Problems). The basis for including a patient in this trial was the written consent of the parents for the child's participation in the study (Protocol № 128 dated 23.12.2019 from the Commission on Bioethics of the Bogomolets NMU).

To verify the genetic deficiency of the folate cycle, the following nucleotide substitutions in the folic acid cycle genes were determined: MTHFR $677 \mathrm{C}>\mathrm{T}$, MTHFR $1298 \mathrm{~A}>\mathrm{C}$, MTRR $66 \mathrm{~A}>\mathrm{G}$ i MTR $2756 \mathrm{~A}>\mathrm{G}$ in various combinations in the homo- and heterozygous state by PCR with restriction. In such children, persistent hyperhomocysteinemia was recognized - a serum concentration of homocysteine above the level of $5.2 \mu \mathrm{mol} / 1$, which is a biomarker of folate cycle deficiency. The number of NK and NKT cells in the blood was measured by laser flow cytofluorimetry (cytometer Epics Xl, USA), using the method of indirect immunofluorescence and monoclonal antibodies to CD markers of lymphocytes (triple label; reagents Beckman Coulter, USA). NK cells meant a subpopulation of lymphocytes with a phenotype CD3CD16+CD56+, and with NKT cells - a subpopulation of lymphocytes with a phenotype $\mathrm{CD} 3+\mathrm{CD} 16+\mathrm{CD} 56+$. At the beginning of the study, NKT-cell deficiency occurred among patients in the study group in $84 \%$ of cases, while NK-cell deficiency - in $51 \%$ of cases, including combined deficiency of NKT-and NK-cells - in $35 \%$ of cases (Figure 1). Immune status studies were performed monthly for 5 consecutive months during a 3-month course of immunotherapy, and for additional 2 months after the completion of immunotherapeutic interventions.

The children of the SG, due to NK- and / or NKT-cell deficiency, received the tested combination immunotherapy. Propes was administered at a 
dose of $2 \mathrm{ml} \mathrm{IM}$, every other day for 3 consecutive months (45 injections). Accordingly, Inflamafertin was administered at a dose of $2 \mathrm{ml} \mathrm{IM}$, every other day for 3 consecutive months, alternating with Propes (45 injections).

The control group (CG) consisted of 32 children of similar age and gender distribution who suffered from autism spectrum disorders associated with genetic deficiency of the folate cycle, but did not receive immunotherapeutic interventions to compensate for the deficiency of NK and NKT cells. These children received only the currently recommended educational development programs in specialized centers for patients with special needs.

Methods of structural and comparative analyzes were used for statistical analysis of the obtained information. In order to establish the reliability of differences in results, the Student's T-test was used to calculate the confidence probability of coefficient $p$ (parametric criterion) and the number of Urbach signs Z (nonparametric criterion). To study the relationship between the assignment of immunotherapy and the dynamics of the studied indicators of immune status, the Pearson Chi-square $(\chi 2)$ was calculated with the determination of the Yates correction. To determine the strength of the detected bonds, the criterion $\varphi$ (F-test), the Pearson correlation coefficient $(\mathrm{C})$ and its normalized value (C') were also calculated. To verify the obtained data, the calculation of the odds ratio (OR) and $95 \%$ confidence interval $(95 \% \mathrm{CI})$ were used. The information was processed using Microsoft Excel.

\section{RESULTS}

The results of structural analysis in the observation groups indicate that the number of NK cells reached the lower limit of norm in 39 out of 53 patients ( $74 \%$ of cases), with an initial deficiency of these lymphocytes. And the average number of NK cells in the blood in SG almost doubled over the 3 months course of immunotherapy, but returned almost to baseline levels in the 2 months after the discontinuation of immunotherapeutic agents. In contrast, the number of NKT cells was normalized in 78 of 87 patients ( $89 \%$ of cases) with an initial deficiency of these cells. Also, the average number of NKT cells in the blood in the SG increased during the course of immunotherapy by at least half and continued to grow steadily for the next 2 months after discontinuation of the tested immunotropic drugs, almost doubling at the end of the observation period.

Data from comparative and variational analyzes indicate a probable difference in the average number of NK cells in the blood in SG and CG during the period of 1-3 months of immunotherapy ( $\mathrm{p}<0.05 ; \mathrm{Z}<\mathrm{Z} 0.05$ ), but not after the discontinuation of immunotherapeutic drugs. A significant difference in the mean numbers of NKT cells in the blood in the observation groups occurred between 2 and 5 months of the study ( $\mathrm{p}<0.05 ; \mathrm{Z}<$ $\mathrm{Z} 0.05)$, but not after the first months of treatment, continuing for at least 2 months following the discontinuation of the tested immunotropic agents (Figures 2 and 3).

The data obtained indicate the ability of combination immunotherapy to increase the number of NK and NKT cells in the blood of children with autism spectrum disorders that are associated with genetic deficiency of the folate cycle, normalizing their immune status. However, the patterns of response from different subpopulations of lympho-

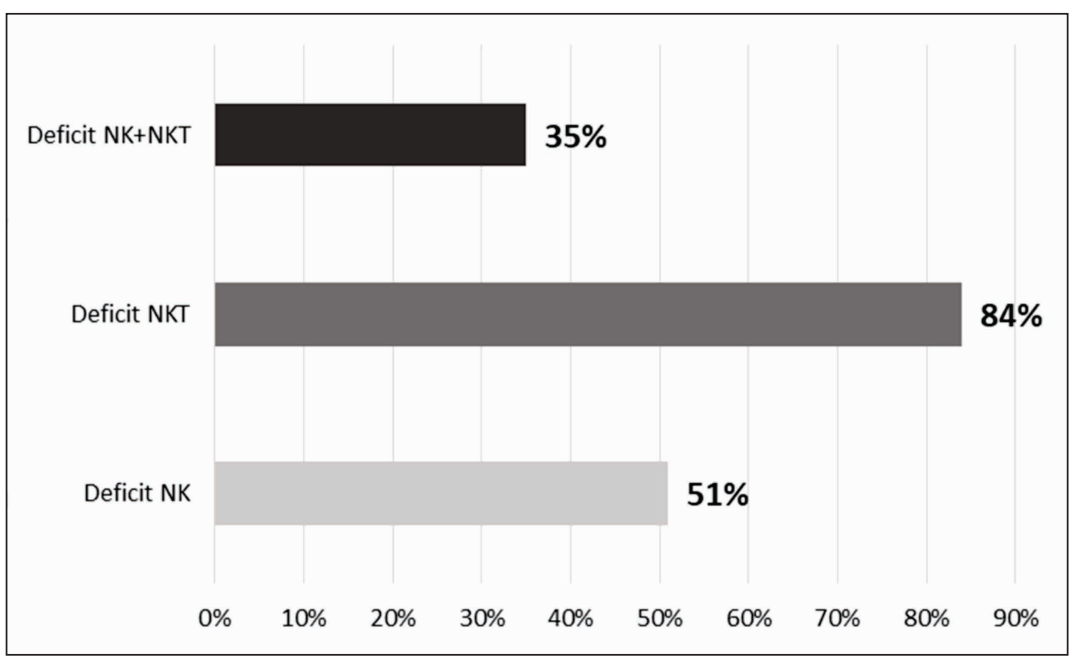

FIGURE 1. The structure of SG $(n=96)$ by deficiencies of NKT- and NK-cells in the blood 


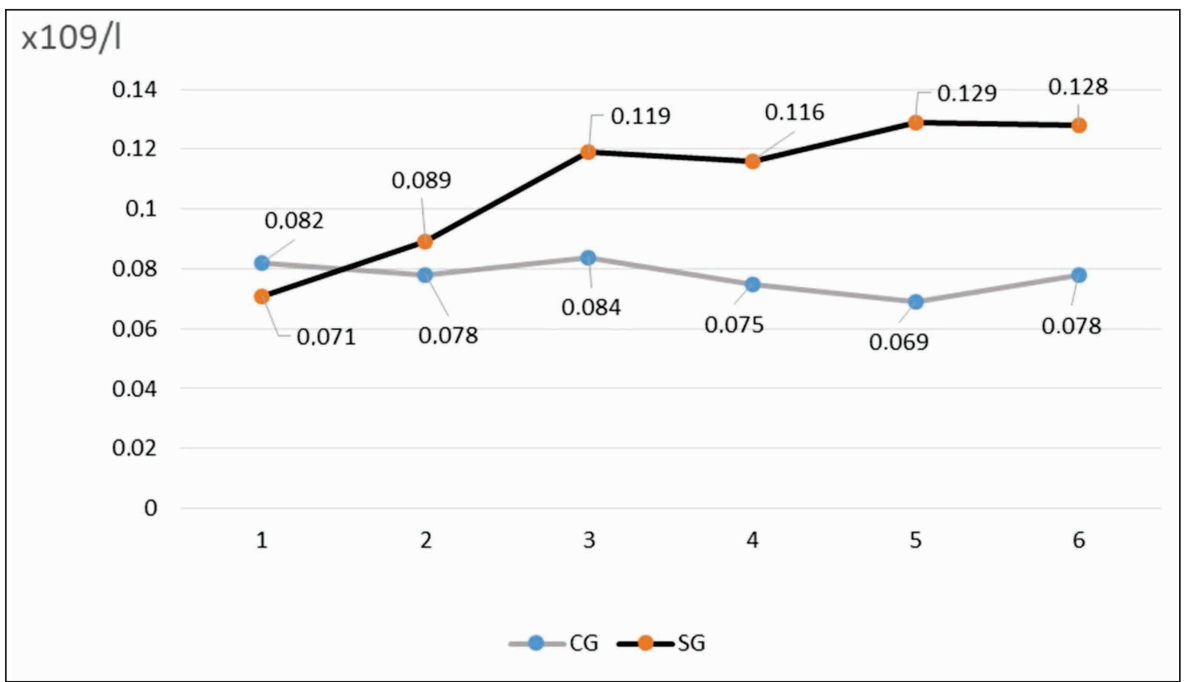

FIGURE 2. Dynamics of the number of NKT cells in the blood in observation groups during the clinical trial

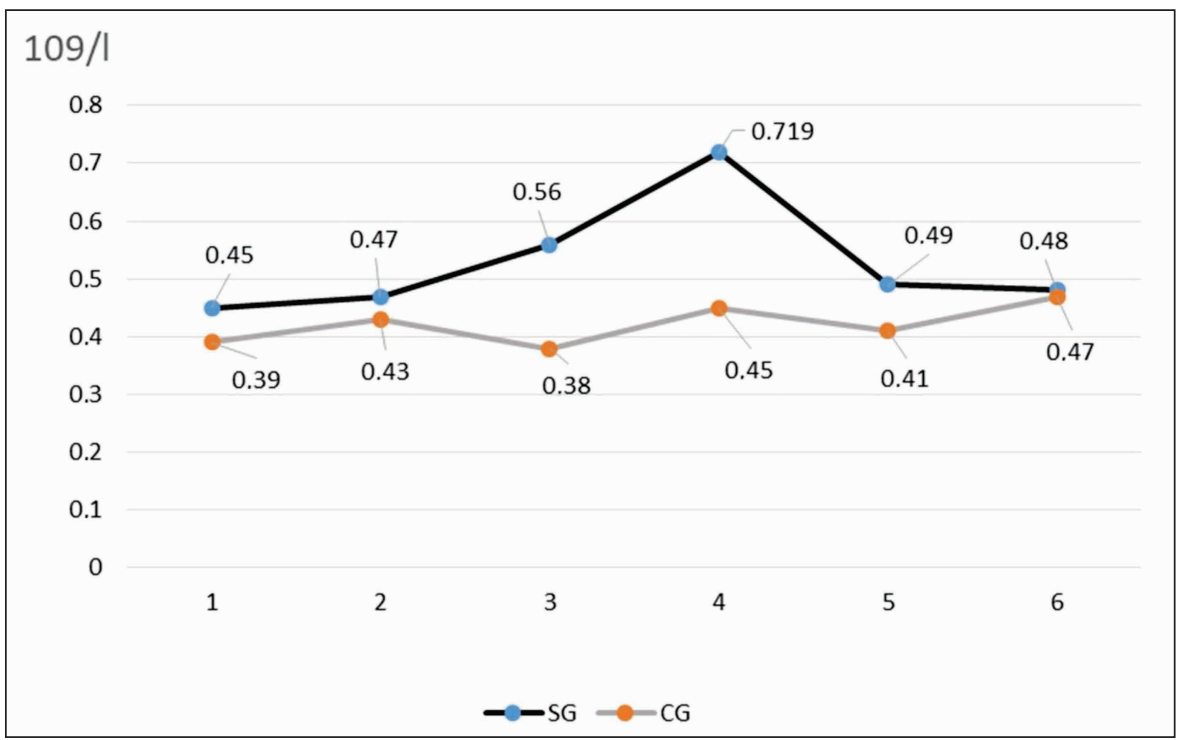

FIGURE 3. Dynamics of the number of NK cells in the blood in observation groups during the clinical trial

cytes to the tested immunotherapeutic agents differ from each other's immune status. Thus, the NK cells respond to immunotherapy faster and more intensely, but the effect is short-lived and lasts only against the backdrop of applied immunotherapy, while the number of NKT cells in the blood grows more slowly with a 1-month delay. However, a prolonged positive effect is achieved, because the gradual increase in the number of these lymphocytes in the blood persists even during the first 2 months after the discontinuation of immunotropic drugs.

To test the association between combination immunotherapy and normalization of the number of NK and NKT cells in the blood, we studied
Pearson's chi-square $(\chi 2)$, the Yates-corrected chisquare test, and the plausibility-adjusted chi-square test. This data would allow to determine whether the applied immunotherapeutic interventions were the cause of the resulting changes in the immune status of SG patients. Based on the data, the number of NKT cells was restored in 78 out of $87 \mathrm{SG}$ patients with initial deficiency of these lymphocytes, and only in 5 out of 32 CG patients with deficiency of these cells before the start of the study. And the number of NK cells reached the norm in 39 out of 53 patients with corresponding deficiency in SG, and only 3 out of 18 people with the initial deficiency in CG. Obtained results are presented in Table 1. 
TABLE 1. Evaluation of chi (X2) Pearson's square and other indicators of contingency between immunotherapy and normalization of immune status in SG patients $(n=96)$

\begin{tabular}{|l|c|c|c|c|}
\hline \multirow{2}{*}{ Indicator } & \multicolumn{2}{|c|}{ NK-cells } & \multicolumn{2}{c|}{ NKT-cells } \\
\cline { 2 - 5 } & Value & Probability & Value & Probability \\
\hline Chi Pearson square & 18.016 & $<0.001$ & 60.65 & $<0.001$ \\
\hline Chi-square with Yates correction & 15.737 & $<0.001$ & 57.307 & $<0.001$ \\
\hline Chi-square adjusted for plausibility & 18.613 & $<0.001$ & 60.282 & $<0.001$ \\
\hline
\end{tabular}

The obtained results (Table 1) indicate a link between immunotherapy and the achievement of normalization of impaired immune status - the number of NK- and NKT-cells in the blood - in children with autism spectrum disorders associated with genetic deficiency of the folate cycle. This indicates that the prescribed immunotropic drugs were the most likely cause of positive changes in the immune status of SG patients.

To study the strength of relationship between the tested immunotherapeutic interventions and the normalization of studied indicators of immune system, the values of the coefficient $\varphi$, the Pearson correlation coefficient and its normalized value were calculated. This would allow us to assess the efficiency of Propes and Inflamafertin on the immune system in children with autism spectrum disorders associated with genetic deficiency of the folate cycle. The obtained results are contained in Table 2.

As can be seen in Table 2, there was a predominantly strong or relatively strong association between immunotherapy and the changes in achieved immune status, indicating a high efficacy of the tested immunotherapeutic agents in the SG. NKT cells were found to be slightly more sensitive to combination immunotherapy than NK lymphocytes, although both subpopulations of lymphocytes showed convincing evidence of a strong association between immunotherapy and normalization of blood counts.

To verify the data on the strong association between immunotherapy and normalization of NK and NKT cell counts in SG patients, an odds ratio
(OR), a standard error of the odds ratio (S), and a $95 \%$ confidence interval $(95 \% \mathrm{CI})$ were calculated. This would avoid errors in assessing the contingency between the studied processes in previous stages of statistical analysis. The obtained results are presented in Table 3.

TABLE 3. Evaluation of odds ratio (OR) and other indicators of the relationship between immunotherapy and normalization of immune status indicators in SG patients $(n=96)$

\begin{tabular}{|l|l|l|}
\hline Indicator & NK-cells & NKT-cells \\
\hline Odds ratio (OR) & 13.929 & 46.800 \\
\hline $\begin{array}{l}\text { Standard error of the } \\
\text { odds ratio (S) }\end{array}$ & 0.705 & 0.601 \\
\hline $\begin{array}{l}\text { 95\% confidence interval } \\
(95 \% \mathrm{Cl})\end{array}$ & $3.498-55.468$ & $14.415-151.937$ \\
\hline
\end{tabular}

As can be seen from Table 3, the calculation of OR and $95 \%$ CI confirms the previously obtained results regarding the close relationship between the tested immunotherapy and normalization of studied indicators of immune status in SG patients. It is a once again demonstrated fact, already identified in the previous stage of statistical data analysis, regarding higher sensitivity of NKT cells compared to NK lymphocytes in the combined immunotherapy of Propes and Inflamafertin in the SG.

\section{DISCUSSION}

The obtained data indicates an appropriate immunomodulatory effect of the tested immunotherapeutic strategy in specific form of immunodeficiency, which is observed in children with autism

TABLE 2. Evaluation of F-test and other indicators of degree of association between immunotherapy and normalization of immune status in SG patients $(n=96)$

\begin{tabular}{|l|l|l|l|l|}
\hline \multirow{2}{*}{ Indicator } & \multicolumn{2}{l|}{ NK-cells } & NKT- cells \\
\cline { 2 - 5 } & Indicator & Degree of Association & Indicator & Degree of_Association \\
\hline $\mathrm{F}^{*}$-criterion & 0.504 & relatively strong & 0.715 & strong \\
\hline Pearson's correlation coefficient (C) & 0.450 & relatively strong & 0.581 & relatively strong \\
\hline $\begin{array}{l}\text { Normalized value of the Pearson } \\
\text { correlation coefficient (C') }\end{array}$ & 0.636 & strong & 0.822 & very strong \\
\hline
\end{tabular}


spectrum disorders associated with genetic deficiency of the folate cycle. Immunodeficiency in children with autism spectrum disorders is most likely responsible for the development of a number of immune-related complications that affect both the severity of mental disorders and the overall health. In particular, it is an abnormally high microbial load on the body [4,5], persistent immunoinflammatory enterocolitis [13], a tendency to generate allergic manifestations [14], systemic inflammation with hypercytokinemia [8,9] and autoimmune reaction against neurons and myelin $[6,7]$. Normalization of impaired immune status is the key to preventing the development of a number of immune-dependent complications in children with autism spectrum disorders, which will improve their clinical condition and can significantly improve the response to neuroprotective therapeutic strategies [15-17].

\section{CONCLUSIONS}

The results obtained in this controlled non-randomized clinical trial indicate that combination immunotherapy with Propes and Inflamafertin is an effective treatment strategy for immunodeficiency caused by genetic folate deficiency in children with autism spectrum disorders. These biological immunotropic drugs are able to normalize the previously reduced number of NK and NKT cells in the blood in this category of patients during a 3-month course of immunotherapy, with a more frequent, stronger and more lasting effect on NKT cells compared to NK lymphocytes.

\section{Funding}

The research was performed as a fragment of scientific work commissioned by the Ministry of Health of Ukraine, grant № 0121U107940.

Conflict of interest: none declared

\section{REFERENCES}

1. Pu D, Shen Y, Wu J. Association between MTHFR gene polymorphisms and the risk of autism spectrum disorders: a meta-analysis. Autism Res. 2013;6(5):384-392.

2. Sadeghiyeh T, Dastgheib SA, Mirzaee-Khoramabadi K, et al. Association of MTHFR $677 \mathrm{C}>\mathrm{T}$ and $1298 \mathrm{~A}>\mathrm{C}$ polymorphisms with susceptibility to autism: A systematic review and meta-analysis. Asian J Psychiatr. 2019;46:54-61.

3. Maltsev DV. Features of folate cycle disorders in children with ASD. Bangladesh J Med Sci. 2020;19(4):737-742.

4. Binstock T. Intra-monocyte pathogens delineate autism subgroups. Med Hypotheses. 2001;56(4):523-531.

5. Nicolson GL, Gan R, Nicolson NL, Haier J. Evidence for Mycoplasma spp., Chlamydia pneumoniae, and human herpes virus- 6 coinfections in the blood of patients with autistic spectrum disorders. J Neurosci Res. 2007 Apr;85(5):1143-8.

6. Cabanlit M, Wills S, Goines P, Ashwood P, Van de Water J. Brain-specific autoantibodies in the plasma of subjects with autistic spectrum disorder. Ann N Y Acad Sci. 2007 Jun;1107:92-103.

7. Frye RE, Sequeira JM, Quadros EV, et al. Cerebral folate receptor autoantibodies in autism spectrum disorder. Mol Psychiatry. 2013;18(3):369-381

8. Masi A, Quintana DS, Glozier N, et al. Cytokine aberrations in autism spectrum disorder: a systematic review and meta-analysis. Mol Psychiatry. 2015;20(4):440-446.

9. Saghazadeh A, Ataeinia B, Keynejad K. A meta-analysis of pro-inflammatory cytokines in autism spectrum disorders: Effects of age, gender, and latitude. J Psychiatr Res. 2019;115:90-102.

10. Maltsev DV. The effectiveness of immunotherapy in the deficiency of natural killers and / or natural killer T lymphocytes in humans. Immunology and Allergology Journal 2016;1:6-7.

11. Maltsev DV. Effectiveness of combined immunotherapy with Propes and Inflamafertin in deficiency of natural corticosteroids and natural killer T-lymphocytes, associated with genetic deficiency of the folate cycle. Immunology and Allergology Journal 2017;1-2:48.

12. He J, Zhang R, Shao M, et al. Efficacy and safety of low-dose IL-2 in the treatment of systemic lupus erythematosus: a randomised, double-blind, placebo-controlled trial. Ann Rheum Dis. 2020;79(1):141-149.

13. Furlano RI, Anthony A, Day R, et al. Colonic CD8 and gamma delta T-cell infiltration with epithelial damage in children with autism. J Pediatr. 2001;138(3):366-372.

14. Xu G, Snetselaar LG, Jing J, Liu B, Strathearn L, Bao W. Association of Food Allergy and Other Allergic Conditions With Autism Spectrum Disorder in Children. JAMA Netw Open. 2018 Jun 1;1(2):e180279.

15. Maltsev D, Natrus L. The Effectiveness of Infliximab in Autism Spectrum Disorders Associated with Folate Cycle Genetic Deficiency. Psychiatry Psychother Clin Psychol. 2020;11(3):583-594.

16. Maltsev DV. Efficiency of a high dose of intravenous immunoglobulin in children with autistic spectrum disorders associated with genetic deficiency of folate cycle enzymes. J Glob Pharma Technol. 2019;11(5):597-609.

17. Maltsev DV. High-dose intravenous immunoglobulin therapy efficiency in children with autism spectrum disorders associated with genetic deficiency of folate cycle enzymes. Psychiatry Psychother Clin Psychol. 2016;2:63-80. 